\title{
Differential Frontal Activation during Exogenous and Endogenous Orientation of Visuospatial Attention
}

\author{
A Near-Infrared Spectroscopy Study \\ Masaharu Takahashi Masanaga Ikegami \\ Department of Psychology, Asahikawa Medical College, Asahikawa, Japan
}

\section{Key Words}

Spatial attention - Near-infrared spectroscopy $\cdot$ Frontal cortex $\cdot$ Visual information processing

\begin{abstract}
Aims: The aim was to measure the prefrontal cortical activity involved in shifting spatial attention to visual stimuli in the left or right visual field using near-infrared spectroscopy. Methods: Eleven participants performed a simple vigilance task, an endogenous attention task and an exogenous attention task. In the endogenous task, the left or the right side of the center diamond brightened, indicating the side for subsequent target appearance (controlled shift of attention). In the exogenous task, the peripheral squares brightened, indicating the appearance of target in those squares (reflexive shift of attention). Results: Stimuli at validly cued locations were responded to faster than stimuli at invalidly cued locations. Increases in oxyhemoglobin (oxy-Hb) were observed in the right prefrontal cortex throughout the 3 tasks, and in the left prefrontal cortex during the vigilance and the exogenous tasks. In the exogenous task, marked decreases in oxy-Hb were observed in the bilateral ventrolateral prefrontal cortex. Conclusions: These results suggest that the left and the right prefrontal cortices mediate distinct cognitive processes during the performance of visuospatial attentional tasks.

Copyright $\odot 2008$ S. Karger AG, Basel
\end{abstract}

\section{Introduction}

Cognitive studies on attention have demonstrated that visual performance in attention tasks is improved or decreased by correct or incorrect cueing of target locations $[1,2]$. In a typical study [1], subjects were instructed to fixate on the center of a screen, where a cue informing them whether the target would be displayed on the right or left side appeared. After a short period, a target appeared on the cued side (valid condition) or on the opposite side (invalid condition). The subjects were required to report the detection of the target as soon as possible. Previous studies have reported that reaction times (RT) were faster in the valid condition than the invalid condition. The effects are thought to reflect our ability to direct attention covertly to a location in extrapersonal space (visuospatial attention).

Neuroimaging studies with positron emission tomography (PET) began to reveal the general architecture of the visuospatial attentional system in the human brain. For example, Corbetta et al. [3] used a visuospatial attentional task, and their PET data indicated that superior parietal cortex, superior frontal cortex in the premotor region, and anterior cingulate gyrus were activated. Nobre et al. [4] also examined the anatomical localizations of the visuospatial attention network using PET. They asked subjects to perform 2 types of visuospatial atten-

\section{KARGER}

Fax +4161306 1234 E-Mail karger@karger.ch www.karger.com
Masaharu Takahashi

Department of Psychology, Asahikawa Medical College

Midorigaoka-higashi 2-1

Asahikawa 078-8510 (Japan)

Tel. +81 16668 2712, Fax +81 16668 2781, E-Mail takam@asahikawa-med.ac.jp 
tional tasks in order to examine whether different activation is observed between reflexive and nonreflexive shifts of visuospatial attention. Both tasks involved covert peripheral shifts of attention directed by peripheral cues (a brief brightening of 1 square). In 1 task, a brief brightening of a square indicated the appearance of the target in the square. This task was supposed to emphasize reflexive aspects of orientation (exogenous attentional shift), since attentional shifts were caused reflexively by the peripheral cues. In the other task, a brief brightening of the square indicated the appearance of the target in the opposite square. This task was supposed to emphasize controlled aspects of orientation (endogenous attentional shift), since subjects had to orient attention to the square that was opposite to the brightened square. Both tasks evoked activations of right anterior cingulate gyrus, intraparietal sulcus of the right posterior parietal cortex, and the medial and lateral premotor cortices.

Recent neuroimaging studies with event-related functional magnetic resonance imaging (ER-fMRI) have begun to isolate cue- and target-related activity in cued attention tasks. For example, Corbetta et al. [5] demonstrated that cues, targets in valid conditions, and targets in invalid conditions evoked different patterns of activation. Cues activate the inferior parietal lobule and superior parietal lobule. On the other hand, targets in valid conditions activate the superior parietal lobule and superior frontal cortex (area at the putative homologue of the frontal eye field), while targets in invalid conditions activate the frontal eye field, superior parietal lobule, and the temporal-parietal junction. They speculated that the intraparietal sulcus is related to voluntary orientation and maintenance of attention at cued locations, and the temporal-parietal junction is related to reorienting attention toward stimuli at unexpected locations [6]. In addition to these regions, it was reported that infrequent or task-irrelevant events activate the ventral frontal cortex (VFC) including the inferior frontal gyrus and middle frontal gyrus $[7,8]$. Based on these results, Corbetta and Shulman [9] proposed 2 networks of brain areas. One system (orienting network or endogenous orienting system) includes the intraparietal sulcus and the frontal eye field, and is responsible for the generation of attentional sets. The other system (reorienting network or exogenous orienting system) includes the temporal-parietal junction and VFC, which are lateralized to the right hemisphere, and is involved in interrupting ongoing cognitive activity when an important stimulus is detected.

The present study used near-infrared spectroscopy (NIRS) to investigate hemodynamic responses in the frontal area of healthy adults during cued attentional tasks. NIRS is a neuroimaging method that measures changes in the oxyhemoglobin (oxy-Hb), deoxyhemoglobin (deoxy-Hb), and total hemoglobin (total-Hb) concentrations in the brain cortex and have increasingly and successfully been employed in functional activation studies in healthy infants and adults. It has a few advantages for visuospatial attention studies compared to fMRI and PET. Since NIRS imposes less physical constraints and subjects can move more naturally than with fMRI and PET [10], it can be applied to infants and children in future developmental studies. In addition, since its signalto-noise ratio is relatively high and a reliable activity can be obtained within a relatively short period of experiments, it can be used to examine brain activities of attention-deficit patients who cannot bear long experiments in clinical studies. The present study used healthy adults and NIRS in order to propose fundamental data on hemodynamic responses in the frontal area during cued attention tasks.

We asked subjects to perform 2 types of visuospatial attentional tasks in order to examine whether endogenous (nonreflexive) and exogenous (reflexive) shifts of visuospatial attention produce different hemodynamic responses in the frontal area. In 1 task, the left or the right side of the center diamond brightened indicating the side for subsequent target appearance (endogenous task). In the other task, the peripheral squares brightened indicating the appearance of target in those squares (exogenous task). The former task was supposed to emphasize controlled aspects of orientation, while the latter was supposed to emphasize reflexive aspects of orientation. As mentioned earlier, Nobre et al. [4] also used 2 types of visuospatial attentional tasks in order to examine reflexive and nonreflexive aspects of attention in their PET study. To speak precisely, however, the 'endogenous task' used by Nobre et al. [4] emphasized both exogenous shifts of attention directed by peripheral cues (a brief brightening of 1 square) and endogenous shifts of attention directed by instructions that a target would appear in the opposite square. The endogenous task adopted in the present experiment, on the other hand, emphasized endogenous shifts of attention to a greater extent than that used in Nobre et al. [4] by excluding exogenous shifts of attention directed by peripheral cues. Based on the 2 networks proposed by Corbetta and Shulman [9], it was expected that the exogenous task would produce greater activations in the right frontal areas than the endogenous task, since the exogenous task activated the exogenous orienting system including the right VFC to a greater degree than the en- 
dogenous task. Since the present study is the first NIRS study to investigate effects of these 2 types of visuospatial attentional tasks on frontal hemodynamics, it was difficult to state more specific hypotheses with regard to expected neural correlates for both tasks.

\section{Participants and Methods}

\section{Participants}

Eleven healthy young adults (Mongoloids; 5 males, 6 females, mean age $=23.7$ years, $S D=5.1$ ) were examined. All participants had normal or corrected-to-normal vision. The handedness of each volunteer was measured by their Edinburgh score, and all participants were right-handed. Participants were paid 1,000 yen per hour. Each subject consented to the experimental procedure, which was approved by the ethics committee of Asahikawa Medical College.

\section{Apparatus}

The experiments were conducted in a small sound-shielded and dimly lit room containing a small desk and a chair. The subject sat at the table on which a notebook computer (IBM ThinkPad T42) was placed. The subject's right hand touched the space key of the computer. Viewing distance was held constant at 50 $\mathrm{cm}$.

\section{Procedure}

Stimuli were displayed onto the computer screen in white, on a black background. The baseline screen consisted of a center diamond $\left(1.2^{\circ}\right.$ wide and $1.2^{\circ}$ high $)$ and two peripheral boxes $\left(1.2^{\circ}\right.$ wide and $1.2^{\circ}$ high), located $10^{\circ}$ apart from the center diamond in a horizontal line. Participants were required to signal the detection of each target by pressing the space key of the computer with the index finger of the dominant hand as quickly as possible.

There were 3 types of cued attention tasks, an endogenous attention task, an exogenous attention task, and a vigilance task (fig. 1).

In the endogenous and exogenous attentional tasks, a trial started with a warning sound (a ping sound of Windows XP), and after $500 \mathrm{~ms}$ a cue was presented in the center diamond (endogenous task) or in the peripheral squares (exogenous task). As a cue, the left or the right side of the diamond (endogenous task), or the left or the right square (exogenous task) brightened for 100 $\mathrm{ms}$, indicating the side of subsequent target appearance. The target stimulus was a cross $(+), 0.6^{\circ}$ wide and $0.6^{\circ}$ high, which appeared in 1 of the peripheral squares. The cue-target interval (stimulus onset asynchrony; SOA) was set at 400 or $800 \mathrm{~ms}$ to reduce temporal orienting. There were 2 trial types with different cueing conditions. In $80 \%$ of the trials, the target appeared on the side indicated by the cue (validly cued trials), and in $20 \%$ of the cases the target appeared on the opposite side (invalidly cued trials). The target remained in the peripheral boxes until the participant pressed a space key or 2,000 ms had elapsed since the onset of the cues. When the participant pressed the space key, an intertrial interval (ITI) started. When 2,000 ms had elapsed without reporting responses from the onset of the cues, the ITI were $0 \mathrm{~ms}$. The length of the ITI varied depending on the length of the SOA (400 or $800 \mathrm{~ms}$ ) and the participant's RT; they varied

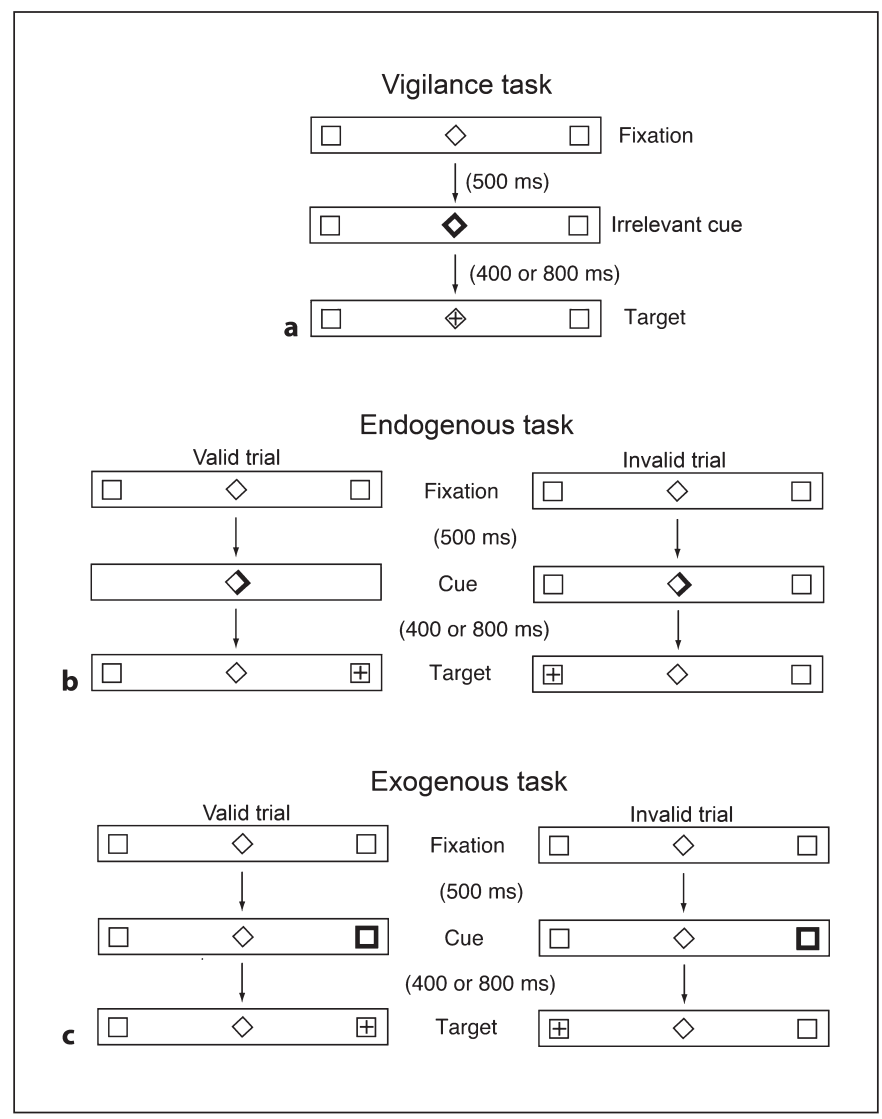

Fig. 1. a Vigilance task: both sides of the diamond brightened and a target always appeared in the center the diamond. b Endogenous task: the left or the right side of the diamond (the right side in this example) brightened and the target $(+)$ appeared on the side indicated by the cue (valid trial) or on the opposite side (invalid trial). c Exogenous task: the left or the right square (the right square in this example) brightened and the target $(+)$ appeared on the side indicated by the cue (valid trial) or on the opposite side (invalid trial).

from 1,200 ms (when the SOA was $800 \mathrm{~ms}$ and the participant's RT was $0 \mathrm{~ms}$ ) to $1,600 \mathrm{~ms}$ (when the SOA was $400 \mathrm{~ms}$ and the RT was $0 \mathrm{~ms}$ ). As a result, the length of the trials was always 2,500 ms. An error was recorded if: (1) a response occurred before target presentation or within $100 \mathrm{~ms}$ after the onset of the target (false alarms); (2) no response occurred by the end of a trial (omissions). The total amount of trials in each block was 20 trials with 16 validly cued trials (8 right- and 8 left-sided targets) and 4 invalidly cued trials ( 2 right- and 2 left-sided targets). Participants were instructed to maintain fixation on the center diamond throughout the experiment, and to detect any peripheral target as quickly as possible. Eye movements were not monitored. Although we cannot rule out the occurrence of eye movements, the likelihood of this possibility might be somewhat diminished by 2 facts. First, participants reported no problems in maintaining fixation. Second, as noted later, patterns of RT obtained in the present study were similar to those obtained from previous studies in which eye 


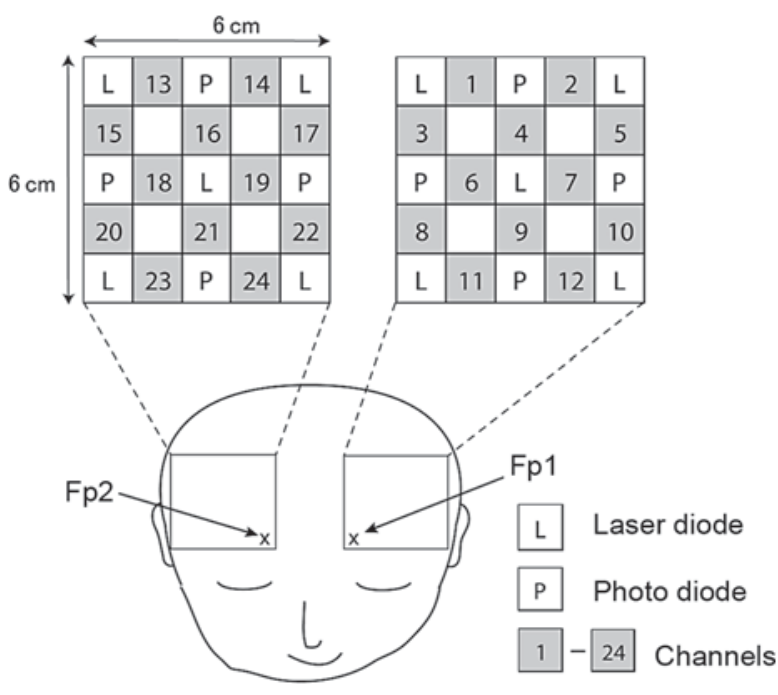

Fig. 2. The positions of the probes (laser diodes and photodiodes) and channels as they were situated on the subject's head. The 2 helmets, each of which was equipped with 5 laser diodes (L; light emitters) and 4 photodiodes (P; light detectors), were located on the left and the right prefrontal regions. The numbers between the diodes indicate channel numbers for the measurement points.

movement were monitored. Before scanning, subjects were informed about the different trial types. They were also told that spatial cues were highly informative, and encouraged to use these cues to improve performance. A 2-min training session was performed before scanning.

The vigilance task was the same as the endogenous task, except that both sides of the diamond brightened and targets appeared in the center diamond. A trial started with a warning sound, and after $500 \mathrm{~ms}$ both sides of the diamond brightened for $100 \mathrm{~ms}$ as an irrelevant cue. The cue did not indicate the side for subsequent target appearance. A target always appeared in the center diamond. The target stimulus was a cross, $0.6^{\circ}$ wide and $0.6^{\circ}$ high. The cue-target interval was 400 or $800 \mathrm{~ms}$. Since cues and targets appeared only in the center diamond, participants did not have to shift their attention at all in this task.

The experiments were conducted in the manner of a blocked design. Each block consisted of a rest period lasting $30 \mathrm{~s}$, a vigilance task lasting $50 \mathrm{~s}$ (20 trials), a rest period lasting $30 \mathrm{~s}$, an endogenous task lasting $50 \mathrm{~s}$ (20 trials), a rest period lasting $30 \mathrm{~s}$, an exogenous attention task lasting $50 \mathrm{~s}$ (20 trials), and a rest period lasting $30 \mathrm{~s}$ (the last rest period corresponded to the first rest period of the next block), in this order. Each participant underwent 3 blocks of experiments.

NIRS Data Acquisition

For near-infrared optical topography, a 24-channel spectrometer operating at 780 and $830 \mathrm{~nm}$ wavelengths (Hitachi ETG-100) was used to measure temporal changes in concentrations of oxy$\mathrm{Hb}$, deoxy-Hb, and total-Hb. A $3 \times 3$ array of 9 probes (5 laser diodes and 4 photodiodes), resulting in 12 channels, was mounted on a plastic helmet (see fig. 2). The helmet covered an area of $6 \times 6 \mathrm{~cm}$. The photodiodes (light detectors) were located $30 \mathrm{~mm}$ from a laser diode (light emitter) and each photodiode detected the reflected near-infrared light of its neighboring/surrounding laser diodes, resulting in 12 links between the emitters and detectors. The laser diodes emitted light of 2 wavelengths (780 and $830 \mathrm{~nm}$ ) modulated at a particular frequency for each wavelength and channel (ranges: 782-793 and 823-833 nm) onto the subject's scalp. The ETG-100 measured data with a sampling rate of $10 \mathrm{~Hz}$. Since changes in the concentration of oxy-Hb and deoxy-Hb depend on the path length of the near-infrared light in the brain, the scale of the hemoglobin quantity is $\mathrm{mmol} \times \mathrm{mm}$. The locations in the international 10-20 system have an appropriate relationship with cortical regions for Mongoloids [11]. Therefore, 2 helmets, each of which included 12 channels $(\mathrm{CH} 1-\mathrm{CH} 12$ in the left helmet, and $\mathrm{CH} 13-\mathrm{CH} 24$ in the right helmet), were placed on the left and right frontal area by referring to the system. As shown in figure 2, the helmet on the right frontal area was placed with its left lower laser diode at Fp2. As a result, the right lower laser diode was located around F8, and the middle upper photo detector was around F4. Similarly, the helmet on the left frontal area was placed with its right lower laser diode at the Fp1. As a result, the left lower laser diode was located around F7, and the middle upper photo detector was around F3. This placement enabled us to measure brain activation in the left brain area among Fp1, F3, and F7, and in the right brain area among Fp2, F4, and F8. According to a probabilistic anatomical platform based on Mongoloids [11], these areas include the anterior, dorsolateral, and ventrolateral prefrontal cortices in both hemispheres.

The ETG-100 system measured changes in the concentration of oxy-Hb, deoxy- $\mathrm{Hb}$, and total-Hb from starting baseline. For the 3 block repetitions, data were averaged according to the task condition (vigilance task, endogenous task, and exogenous task) with a 6-second 'baseline' preceding each task period and a 6-second 'rest' segment following each task block. The block was divided into a 6second pretask baseline period (pre-task period), a 50-second task period, a 12-second relaxing period (relaxing period), and a 6-second posttask baseline period (posttask period). The 12-second relaxing period was introduced since our previous studies indicated that changes in the $\mathrm{Hb}$ concentrations ended within $12 \mathrm{~s}$ after the tasks. This delay is consistent with previous reports of hemodynamic responses in the cortex $[12,13]$. Spontaneous cerebral blood flow oscillation, which originates through vasomotion of large cerebral arteries, and whole body blood flow changes, which synchronize over the whole channel array, were corrected by a baseline correction of a linear fitting performed on the data between the 6second pretask and 6-second posttask periods. For statistical analyses, the mean values of each of the time segments for the 3 attention tasks were calculated for each participant and NIRS channel.

\section{Results}

\section{Behavioral Data}

Table 1 presents the mean RT to different trial types and tasks. For the vigilance task, a 1-way repeated-mea- 
Table 1. RT for different trial types

\begin{tabular}{lllllll}
\hline Trial type & \multicolumn{2}{l}{ Mean RT, ms } & & & & \\
\cline { 2 - 7 } & vigilance & $\mathrm{p}$ & endogenous & $\mathrm{p}$ & exogenous & $\mathrm{p}$ \\
\hline $400-\mathrm{ms} \mathrm{SOA}$ & $318 \pm 10$ & $<0.001$ & $368 \pm 11$ & & $374 \pm 10$ & \\
$800-\mathrm{ms} \mathrm{SOA}$ & $287 \pm 10$ & & $339 \pm 15$ & & $352 \pm 14$ & $<0.05$ \\
\hline Valid cue & - & $316 \pm 6$ & $<0.001$ & $344 \pm 8$ & \\
Invalid cue & - & $391 \pm 17$ & & $381 \pm 15$ & $<0.1$ \\
\hline Left VFC & - & $355 \pm 12$ & n.s. & $369 \pm 13$ & n.s. \\
Right VFC & - & $352 \pm 16$ & & $356 \pm 11$ & \\
\hline
\end{tabular}

Values are given as means \pm SD. n.s. $=$ Not significant.

sures analysis of variance (ANOVA) was conducted with SOA (400 vs. $800 \mathrm{~ms}$ ) as a factor. There was a significant effect of SOA in the vigilance task $[\mathrm{F}(1,10)=99.89$, $\mathrm{p}<$ $0.001]$. For endogenous and exogenous tasks, on the other hand, a 3-way repeated-measures ANOVA was conducted with SOA (400 vs. $800 \mathrm{~ms}$ ), cues (valid vs. invalid), and visual fields (right vs. left) as factors. There was no significant effect of visual fields on RT in both tasks. There was a tendency toward significance for SOA in the endogenous task $[\mathrm{F}(1,10)=4.42, \mathrm{p}=0.006]$. There was a significant effect of $\mathrm{SOA}$ in the exogenous task $[\mathrm{F}(1,10)=$ $5.74, \mathrm{p}<0.05]$. There was a significant effect of cues (valid vs. invalid) in the endogenous task $[\mathrm{F}(1,10)=25.46$, $\mathrm{p}<0.001]$. There was a tendency toward significance for cues in the exogenous task $[\mathrm{F}(1,10)=3.92, \mathrm{p}=0.08]$. There was no interaction between the 3 factors. Based on these results, a 3-way repeated-measures ANOVA was additionally conducted with SOA, cues, and tasks (endogenous vs. exogenous tasks) as factors for data obtained from the endogenous and the exogenous tasks. There were significant effects of SOA $[\mathrm{F}(1,10)=8.30, \mathrm{p}<0.05]$ and cues $[\mathrm{F}(1,10)=18.55, \mathrm{p}<0.01]$ on RT. However, there was no significant effects of task factor (endogenous vs. exogenous tasks) on RT $[\mathrm{F}(1,10)=0.72, \mathrm{p}=0.42]$. No interactions were significant.

In order to examine the effects of tasks on error responses, mean frequencies of false alarms and omission errors per 60 trials ( 3 blocks) were computed for the 3 tasks. Mean frequencies of false alarms were 1.12 in the vigilance task, 0.91 in the endogenous task and 1.18 in the exogenous task and a 1-way repeated-measures ANOVA revealed that there was no significant difference among the 3 tasks $[F(2,20)=0.13, p=0.88]$. On the other hand, mean frequencies of omissions were 0.46 in the vigilance task, 0.27 in the endogenous task and 1.00 in the exogenous task and a 1-way repeated-measures ANOVA revealed that there was tendency toward significance among the 3 tasks $[\mathrm{F}(2,20)=2.74, \mathrm{p}=0.09]$.

The endogenous task and the exogenous tasks have the same task structures, except for different cueing conditions. In order to examine effects of the 2 types of cuing conditions on error responses, a 1-way repeated-measures ANOVA was conducted for error responses in the endogenous and exogenous tasks. There was no significant difference between false alarms in the endogenous and the exogenous tasks $[\mathrm{F}(1,10)=2.61, \mathrm{p}=0.16]$. However, there was a significant difference between omissions in the endogenous and the exogenous tasks $[\mathrm{F}(1,10)=$ 7.11, $\mathrm{p}<0.05]$.

\section{NIRS Data}

Although recent simultaneous fMRI-NIRS measurement studies [14, 15] have suggested that oxy- $\mathrm{Hb}$ is strongly correlated with the fMRI signal, there is still controversy as to the choice between oxy- and deoxy- $\mathrm{Hb}$ as an appropriate index of hemodynamic response to cognitive processes [16]. Therefore, we focused on both oxy- and deoxy-Hb. We observed marked oxy-Hb increases and decreases in some channels during the task period. Figure 3 shows changes of averaged oxy-Hb and deoxy-Hb quantities $(\mathrm{mmol} \times \mathrm{mm})$ in 2 representative channels in which oxy-Hb significantly increased (left panel) or decreased (right panel). Since marked increases or decreases in oxy-Hb were observed shortly after the onset of the task period (gray bar on the $\mathrm{x}$-axis) and they gradually returned to the baseline during the rest period, these responses were considered to be task-induced.

Five of 24 channels $(\mathrm{CH} 1, \mathrm{CH} 2, \mathrm{CH} 3, \mathrm{CH} 14$, and $\mathrm{CH} 17)$, which were located in the upper area of the probes, were affected by severe measurement noise in 1-4 subjects. Because of the within-subject nature of comparison in the present experimental design, data obtained from 
Fig. 3. Time course of averaged oxy-Hb and deoxy-Hb quantities ( $\mathrm{mmol} \times \mathrm{mm}$ ) in a representative channels in which significant increments (a; $\mathrm{CH} 22$ ) and decrements (b; CH10) in oxy-Hb were observed in the exogenous task. The data were analyzed with the integral mode and were averaged across 11 participants. The error bars represent standard deviations.

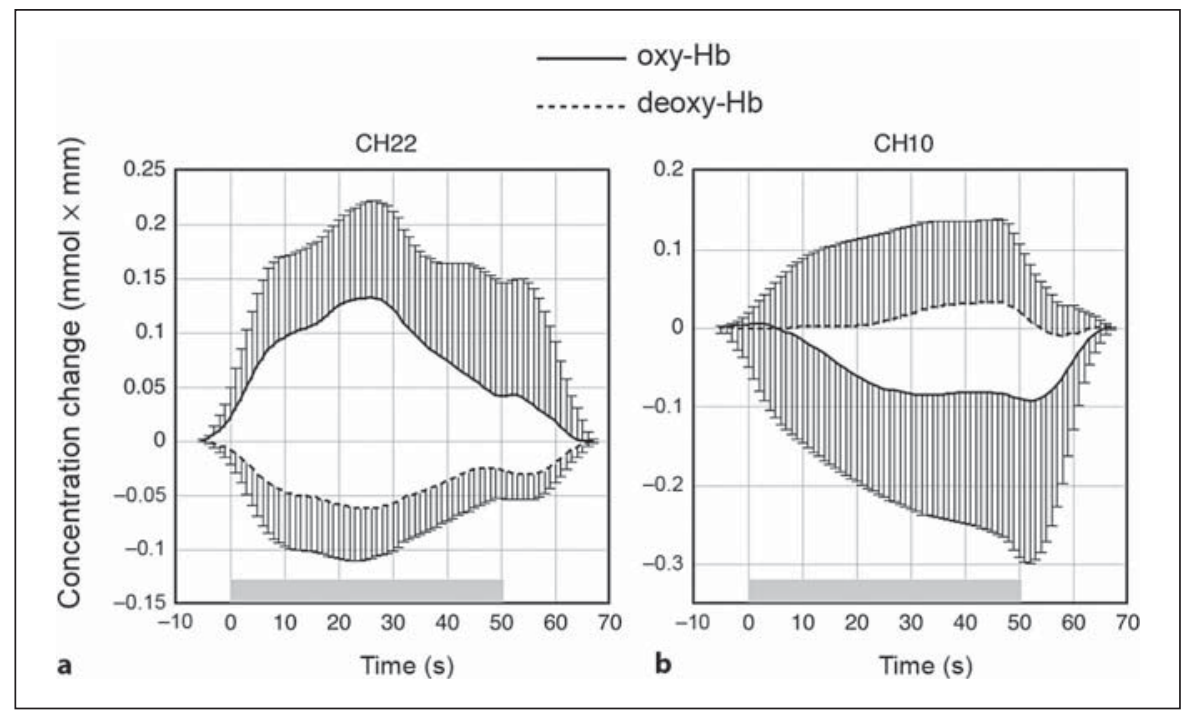

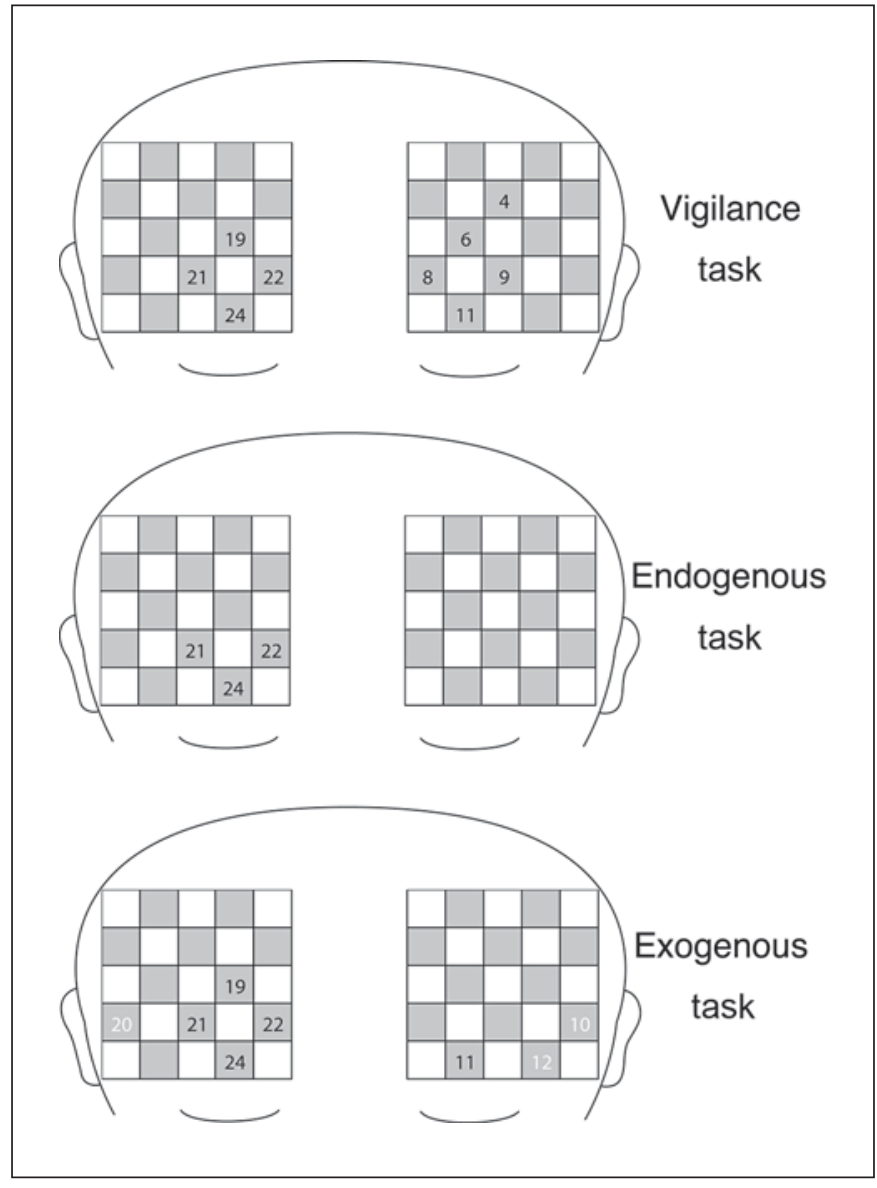

Fig. 4. Schematic diagram of the positions of the channels that showed significant changes in oxy-Hb during the task periods. The numbers indicate channels that showed significant increments (black) or decrements (white). these 5 channels were excluded from the following analyses. As a result, a 3-way repeated measures ANOVA was conducted for the oxy-Hb data to determine the effects of manipulating tasks (vigilance, endogenous, and exogenous), channels (CH4-13, CH15-16, and $\mathrm{CH} 18-24)$, and time (pretask period and task period) on the hemodynamic responses. There was a significant main effect of channels $[\mathrm{F}(18,180)=4.68, \mathrm{p}<0.001]$ and a significant main effect of time $[\mathrm{F}(1,10)=8.76, \mathrm{p}<0.05]$. On the other hand, a main effect for tasks was not significant $[\mathrm{F}(2$, $10)=1.96, p=0.16]$. There was a significant tasks $\times$ channels interaction $[\mathrm{F}(36,360)=1.52, \mathrm{p}<0.05]$, a significant tasks $\times$ time interaction $[\mathrm{F}(2,20)=4.04, \mathrm{p}<$ $0.05]$, and a significant channels $\times$ time interaction $[\mathrm{F}(18$, $180)=5.64, p<0.001]$. In addition, there was a significant tasks $\times$ channels $\times$ time interaction $[\mathrm{F}(36,360)=1.49$, $\mathrm{p}<0.05]$. As we were interested in differences between tasks and channels, we performed post hoc tests using Tukey HSD (honestly significant difference) to investigate the precise tasks and channels in which task periods produced significant changes in oxy-Hb. Table 2 summarizes channels in which statistically significant oxy- $\mathrm{Hb}$ increments (+) or decrements (-) were observed between the pretask period and the task period. Figure 4 is a schematic diagram of the positions of the channels that showed significant increases or decreases in oxy- $\mathrm{Hb}$ in the task periods for the 3 tasks. The task period produced significant increases in oxy-Hb in: 5 channels in the left hemisphere $(\mathrm{CH} 4, \mathrm{CH} 6, \mathrm{CH} 8, \mathrm{CH} 9$, and $\mathrm{CH} 11)$ and 4 channels in the right hemisphere $(\mathrm{CH} 19, \mathrm{CH} 21, \mathrm{CH} 22$, and $\mathrm{CH} 24$ ) during the vigilance task; 3 channels in the 
Table 2. Channels that showed significant oxy-Hb changes in response to the task periods (Tukey's HSD, $\mathrm{p}<0.05$ )

\begin{tabular}{llll}
\hline $\begin{array}{l}\text { Hemi- } \\
\text { sphere }\end{array}$ & \multicolumn{2}{l}{ Task } & \\
\cline { 2 - 4 } & vigilance & endogenous & exogenous \\
\hline Left & $\mathrm{CH} 4(+0.051)$ & & $\mathrm{CH} 10(-0.060)$ \\
& $\mathrm{CH} 6(+0.065)$ & & $\mathrm{CH} 11(+0.044)$ \\
& $\mathrm{CH} 8(+0.078)$ & & $\mathrm{CH} 12(-0.058)$ \\
& $\mathrm{CH} 9(+0.069)$ & & \\
\hline Right & $\mathrm{CH} 11(+0.089)$ & & $\mathrm{CH} 19(+0.052)$ \\
& $\mathrm{CH} 19(+0.058)$ & $\mathrm{CH} 21(+0.046)$ & $\mathrm{CH} 20(-0.048)$ \\
& $\mathrm{CH} 22(+0.092)$ & $\mathrm{CH} 22(+0.059)$ & $\mathrm{CH} 21(+0.046)$ \\
& $\mathrm{CH} 24(+0.114)$ & $\mathrm{CH} 24(+0.050)$ & $\mathrm{CH} 22(+0.083)$ \\
& & & $\mathrm{CH} 24(+0.067)$ \\
& & &
\end{tabular}

The significant increments (+) and decrements (-) in oxy-Hb $(\mathrm{mmol} \times \mathrm{mm})$ between the pre-task period and task are shown in parentheses.

right hemisphere ( $\mathrm{CH} 21, \mathrm{CH} 22$, and $\mathrm{CH} 24)$ in the endogenous task; 1 channel in the left hemisphere $(\mathrm{CH} 11)$ and 4 channels in the right hemisphere (CH19, $\mathrm{CH} 21, \mathrm{CH} 22$ and $\mathrm{CH} 24$ ) during the exogenous task. In addition, the task periods produced significant decreases in oxy- $\mathrm{Hb}$ in 2 channels in the left hemisphere ( $\mathrm{CH} 10$ and $\mathrm{CH} 12)$ and 1 channel in the right hemisphere $(\mathrm{CH} 20)$ in the exogenous task. Channels in which increases in oxy-Hb were observed were located near Fp1 and Fp2, whereas channels in which decreases in oxy-Hb were observed were located in near Fp7 and Fp8. Additionally, we performed post hoc tests using Tukey HSD to analyze whether the oxy-Hb data are statistically different between tasks for the channels. In the task periods of $\mathrm{CH} 12$, the oxy- $\mathrm{Hb}$ data in the exogenous task were significantly smaller than those in the vigilance and the endogenous tasks. In the task periods of $\mathrm{CH} 20$, the oxy- $\mathrm{Hb}$ in the exogenous task was significantly smaller than that in the endogenous task.

A 3-way repeated measures ANOVA was conducted for the deoxy-Hb data to determine the effects of manipulating tasks, channels, and time on the hemodynamic responses. There was a significant main effect for channels $[\mathrm{F}(18,180)=2.66, \mathrm{p}<0.01]$, indicating a significant difference across channels. On the other hand, a main effect for tasks was not significant $[F(2,20)=1.41, p=0.27]$, and a main effect for time was not significant $[F(1,10)=$ $3.81, \mathrm{p}=0.08]$. There was a significant channels $\times$ time interaction $[\mathrm{F}(18,180)=2.56, \mathrm{p}<0.01]$. No other interac- tions were significant. Post hoc tests using Tukey HSD were performed to investigate the precise channels in which task periods produced significant changes in deoxy-Hb. The task period produced significant decreases in deoxy-Hb in $\mathrm{CH} 11, \mathrm{CH} 22$ and $\mathrm{CH} 24$.

\section{Discussion}

\section{Behavioral Data}

For combined data obtained from endogenous and exogenous tasks, there were significant effects of SOA and cues on RT. Therefore, it was suggested that RT were shorter in the valid condition than in the invalid condition, and were shorter for the long SOA than for the short SOA. These results were consistent with those obtained in previous studies $[1,2]$, suggesting that subjects effectively used the cues regarding the location of the target. For difficulty of different cueing conditions (endogenous vs. exogenous tasks), there was no significant effect of conditions (endogenous vs. exogenous tasks) on RT. In addition, there were no significant effects of tasks on mean frequencies of false alarms between these cueing conditions. However, omissions were more frequent in the exogenous task than in the endogenous task. Therefore, it was suggested that the exogenous task was more difficult than the endogenous task.

\section{NIRS Data}

The 3 tasks produced differential patterns of increases in oxy-Hb in the left and the right prefrontal areas. For the right hemisphere, 3 channels in the right hemisphere $(\mathrm{CH} 21, \mathrm{CH} 22$, and $\mathrm{CH} 24)$ produced activations (increases in oxy-Hb) throughout the 3 tasks. For the left hemisphere, on the other hand, activations were observed in 5 channels in the left hemisphere during the vigilance task (CH4, $\mathrm{CH} 6, \mathrm{CH} 8, \mathrm{CH} 9$, and $\mathrm{CH} 11)$ and 1 channel in the left hemisphere during the exogenous tasks (CH11), whereas no activation was produced during the endogenous task. For deoxy-Hb, decreases were observed only in 3 channels (CH11, $\mathrm{CH} 22$, and $\mathrm{CH} 24$ ) throughout the 3 tasks. Thus, this discussion focuses on oxy-Hb.

The right prefrontal activations were common among the 3 tasks. The 2 networks proposed by Corbetta and Shulman [9] predicted that the exogenous task would produce greater activations in the right frontal areas, because the exogenous orienting system in their model includes VFC in the right hemisphere. In the present study, however, the right prefrontal activations were also ob- 
served in the vigilance task, which did not require exogenous orienting of visuospatial attention. In addition, the activated areas in the present study $(\mathrm{CH} 21, \mathrm{CH} 22$, and $\mathrm{CH} 24$ ) seemed to be located more rostrally than those included in the exogenous orienting system [7-9]. Therefore, the present results are not in line with the model proposed by Corbetta and Shulman [9].

Alternatively, the 3 tasks in the present study had a common task feature of response selection (choosing responses given environmental stimuli). Therefore, it is plausible that the right frontal activations in the present study might be related to cognitive processes of response selection. Neuroimaging research on neural substrates of response selection with fMRI has focused primarily on the anterior prefrontal cortex, dorsolateral prefrontal cortex, and ventrolateral prefrontal cortex [17]. Some of the research suggests that the right prefrontal cortex mediates spatial response selection for spatial materials [17-22], whereas other research suggests that the left prefrontal cortex is activated by response selection [2325]. The activation of the right prefrontal cortex observed throughout the 3 tasks in the present NIRS study seems to provide additional support for the hypothesis that the right prefrontal cortex may mediate response selection for spatial materials [17].

For the left prefrontal cortex, increases in oxy-Hb were observed in 5 channels during the vigilance task and in 1 channel during the exogenous task. Unfortunately, the functions of the left prefrontal cortex are controversial at the present $[17,26]$. As mentioned earlier, practice-related increases in activations were reported in the left prefrontal cortex with a response selection task, suggesting that the left prefrontal cortex mediates response selection [25]. In the present study, however, the activations observed in the left hemisphere were unlikely to be related to response selection since the activations were not observed consistently across the 3 tasks. On the other hand, a variety of studies have suggested that activations in the prefrontal cortex may be attributed to thought processes that are task unrelated and stimulus independent, since the activations in the prefrontal cortex were observed in a variety of tasks that are supposed to involve self-referential mental processes [27-31]. Recently, however, it was demonstrated that performance-related activations were observed in the left prefrontal cortex during a simple RT task, suggesting that the left prefrontal cortex played a role in maintaining attention toward the external environment, but not in generating any information internally [26]. Moreover, it was reported that activity in this region was related to the degree of visual attention to ex- ternal stimuli in the exogenous type of visuospatial task [32]. In the present study, the activations in the left prefrontal area appeared during the vigilance and the exogenous tasks. These results were consistent with the hypothesis that the left prefrontal cortex promotes engagement with the external environment $[26,32]$. On the other hand, there was no activation in the left prefrontal cortex during the endogenous task. The reasons for this discrepancy are unknown at the present. However, one tentative hypothesis is that compared to the vigilance and the exogenous tasks the endogenous task may include an additional cognitive process of endogenous shifts of visuospatial attention (top-down control of attention) depending on cues. It is plausible that this process attenuated the degree to which participants engage with external stimuli in the endogenous task.

In the exogenous task, 2 channels around F7 (CH10, and $\mathrm{CH} 12)$ and 1 channel around F8 (CH20) produced significant decreases in oxy-Hb during the task period. These channels seemed to be located in the middle or the inferior frontal gyri in both hemispheres [11]. There are several NIRS studies which found significant decreases in oxy- and total-Hb. For example, Wenzel et al. [33] found decreases in oxy- and total-Hb in the occipital cortex. Shimada et al. [10] also reported significant decreases in oxyand total-Hb in the prefrontal area during reaching tasks. In addition, decreases in oxy- and total- $\mathrm{Hb}$ have been reported in clinical studies. Hock et al. [34] reported a significant decrease in oxy- and total-Hb during performance of a verbal fluency task in patients with Alzheimer's disease. Similarly, Sakatani et al. [35] found a significant decrease in oxy- and total- $\mathrm{Hb}$ during electrical stimulation to the thalamus in patients with Parkinson's disease.

Although the whole mechanism of decreases in oxyand total-Hb remains unresolved, simultaneous fMRINIRS measurement studies [14, 15] have suggested that oxy-Hb is strongly correlated with the fMRI signal, and the decrease in oxy-Hb observed in NIRS measurement is essentially the same hemodynamic processes as the deactivation seen in fMRI and PET studies [10]. Cerebral neuron activation causes an increase in oxy-Hb consumption and cerebral vasodilatation, and, subsequently, cerebral blood volume begins to increase. It has been suggested that since cerebral blood flow exceeds the concomitant local increase in tissue metabolic rate, the typical response is an increase in oxy- $\mathrm{Hb}$ and a decrease in deoxy- $\mathrm{Hb}$. As a result, the decreases in oxy- and total- $\mathrm{Hb}$ are thought to represent the deactivation of cognitive functions [10]. On the other hand, it is possible that if cerebral blood flow does not exceed the local increase in tissue metabolic rate, 
the typical response is a decrease in oxy- $\mathrm{Hb}$ and an increase in deoxy-Hb [36]. In fact, it was reported that healthy subjects showed a decrease in cerebral blood flow in the frontal lobe during problem solving and mental arithmetic [37]. Thus, there is a possibility that the decreases in oxy- and total-Hb may represent overactivation. Clearly, the relationship between neural activation and hemodynamic response is more complicated [38].

If the decreases in oxy- and total-Hb are thought to represent overactivation of corresponding areas, the present results could be interpreted as suggesting that the prefrontal cortex was overactivated during the exogenous task. As mentioned earlier, one aspect of the exogenous task is to require the participants to inhibit top-down control of attentional orientation and engage with external stimuli in a wide area to a greater degree than the endogenous task. Therefore, this interpretation of the present result would be in line with the findings that the ventrolateral prefrontal cortex activated when participants were required to inhibit controlled shifts of attentional orientation in the continuous performance task $[9,39]$.

On the other hand, there is a possibility that the decreases in oxy-Hb could represent deactivation of the corresponding areas. If this is the case in the present study, possible explanations should be discussed for the deactivation observed in the present exogenous task. First, during the cue period, the exogenous task required subjects to watch occurrences of cues in a wide area, whereas the vigilance and endogenous tasks required subjects to show selective attention to the center cues [2]. In other words, subjects had to exhibit active focal attention based on the cues in the vigilance and endogenous tasks, whereas they had to reduce focal attention and use their passive attentional processing triggered by peripheral cues in the exogenous task (preattentive processing, Neisser [40]). Therefore, the deactivation observed in the exogenous task may reflect suppression of active focal attention. However, this speculation seems implausible for the present results, since deactivated areas in the exogenous task were not activated by the vigilance and the endogenous tasks that required subjects to show focal attention. Second, it has been suggested that deactivation in dorsal prefrontal areas may be related to task difficulty or attention demand in a variety of cognitive tasks [10, 41-43]. If the prefrontal area is activated during the rest period, it should be relatively deactivated during an attention-demanding task. This interpretation is consistent with the present behavioral data, which indicated that the mean frequency of omission in the exogenous task was significantly greater than that in the endogenous task, suggest- ing that the former task may be more attention-demanding than the latter task.

There are limitations that have to be considered in the interpretation of the present results. First, there is a possibility that the difference in obtained oxy- and deoxy- $\mathrm{Hb}$ concentrations does not reflect a functional difference, but a structural difference [44]. In other words, path lengths may vary among participants, which suggests that direct comparison of individual NIRS data might be inappropriate [39]. Second, the present study adopted a block design, which made it difficult to examine hemodynamic responses for each single trial $[45,46]$. Clearly, future studies should adopt an event-related design. Third, the present study examined only a relatively small number of participants. Thus, it is preferable to conduct a future study with a larger sample size. Despite these limitations, our results demonstrated differential frontal activation during exogenous and endogenous orientation of visuospatial attention. The hemispheric dissociation between the activations in the frontal cortex during spatial attention suggests that these regions recruit distinct cognitive processes [17]. Further research is needed, which identifies more specific conditions under which activation and deactivation are observed in the frontal cortex. This effect will have to be considered in future network models of visuospatial attention.

\section{Acknowledgments}

This work was supported by grants $(13224002,13610075)$ from the Japanese Ministry of Education, Culture, Sports, Science and Technology, and a grant (Information and Human Activity, Precursory Research for Embryonic Science and Technology) from the Japan Science and Technology Corporation.

References

$\checkmark 1$ Posner MI: Orienting of attention. Q J Exp Psychol 1980;32:3-25.

2 Posner MI, Cohen Y: Components of performance; in Bouma H, Bowhuis D (eds): Attention and Performance. Hillsdale, Erlbaum, 1984, pp 531-556.

-3 Corbetta M, Miezin FM, Shulman GL, Petersen SE: A PET study of visuospatial attention. J Neurosci 1993;13:1202-1226.

-4 Nobre AC, Sebestyen GN, Gitelman DR, Mesulam MM, Frackowiak RS, Frith CD: Functional localization of the system for visuospatial attention using positron emission tomography. Brain 1997;120:515-533.

5 Corbetta M, Kincade JM, Ollinger JM, McAvoy MP, Gordon L, Shulman GL: Voluntary orienting is dissociated from target detection in human posterior parietal cortex. Nat Neurosci 2000;3:292-297. 
6 Tootell RBH, Hadjikhani N: Attention brains at work! Nat Neurosci 2000;3:206208.

7 Braver TS, Barch DM, Gray JR, Molfese DL, Snyder A: Anterior cingulate cortex and response conflict: effects of frequency, inhibition and errors. Cereb Cortex 2001;11:825836.

$\checkmark 8$ Downar J, Crawley AP, Mikulis DJ, Davis KD: The effect of task relevance on the corti$\mathrm{cal}$ response to changes in visual and auditory stimuli: an event-related fMRI study. Neuroimage 2001;14:1256-1267.

-9 Corbetta M, Shulman GL: Control of goaldirected and stimulus-driven attention in the brain. Nat Rev Neurosci 2002;3:201-215.

10 Shimada S, Hiraki K, Matsuda G, Oda I: Decrease in prefrontal hemoglobin oxygenation during reaching tasks with delayed visual feedback: a near-infrared spectroscopy study. Cogn Brain Res 2004;20:480-490.

- 11 Okamoto M, Dan H, Sakamoto K, Takeo K, Shimizu K, Kohno S, Oda I, Isobe S, Suzuki T, Kohyama K, Dan I: Three-dimensional probabilistic anatomical cranio-cerebral correlation via the international $10-20$ system oriented for transcranial functional brain mapping. Neuroimage 2004;21:99-111.

-12 Ehlis AC, Herrmann MJ, Wagener A, Fallgatter AJ: Multi-channel near-infrared spectroscopy detects specific inferior-frontal activation during incongruent Stroop trials. Biol Psychol 2005;69:315-331.

13 Fallgatter AJ, Strik WK: Right frontal activation during the continuous performance test assessed with near-infrared spectroscopy in healthy subjects. Neurosci Lett 1997;223:8992.

- 14 Strangman G, Culver JP, Thompson JH, Boas DA: A quantitative comparison of simultaneous bold fMRI and NIRS recordings during functional brain activation. Neuroimage 2002;17:719-731.

15 Yamamoto T, Kato T: Paradoxical correlation between signal in functional magnetic resonance imaging and deoxygenated haemoglobin content in capillaries: a new theoretical explanation. Phys Med Biol 2002;47: 1121-1141.

16 Obrig H, Wenzel R, Kohl M, Horst S, Wobst P, Steinbrink J, Thomas F, Villringer A: Near-infrared spectroscopy: does it function in functional activation studies of the adult brain? Int J Psychophysiol 2000;35:125-142.

17 Schumacher EH, Hendricks MJ, D'Esposito: Sustained involvement of a frontal-parietal network for spatial response selection with practice of a spatial choice-reaction task. Neuropsychologia 2005;43:1444-1455.

18 Dassonville P, Lewis SM, Zhu XH, Ugurbil K, Kim SG, Ashe J: The effect of stimulusresponse compatibility on cortical motor activation. Neuroimage 2001;13:1-14.

19 Jiang Y, Kanwisher N: Common neural mechanisms for response selection and perceptual processing. J Cogn Neurosci 2003; 15:1095-1110.
20 Merriam EP, Colby CL, Thulborn KR, Luna B, Olson CR, Weeney JA: Stimulus-response incompatibility activates cortex proximate to three eye fields. Neuroimage 2001;13:794800.

21 Schumacher EH, D’Esposito M: Neural implementation of response selection in humans as revealed by localized effects of stimulus-response compatibility on brain activation. Hum Brain Mapp 2002;17:193-201.

22 Schumacher EH, Elston PA, D’Esposito M: Neural evidence for representation-specific response selection. J Cogn Neurosci 2003;15: 1111-1121.

23 Bunge AS, Kahn I, Wallis JD, Miller EK, Wagner AD: Neural circuits subserving the retrieval and maintenance of abstract rules. J Neurophysiol 2003;90:3419-3428.

24 Bunge SA: How we use rules to select actions: a review of evidence from cognitive neuroscience. Cogn Affect Behav Neurosci 2004;4: 564-579.

25 Iacoboni M, Woods RP, Mazziotta JC: Brainbehavior relationships: evidence from practice effects in spatial stimulus-response compatibility. J Neurophysiol 1996;76:321331.

26 Gilbert SJ, Simons JS, Frith CD, Burgess PQ: Performance-related activity in medial rostral prefrontal cortex (area 10) during lowdemand tasks. J Exp Psychol 2006;32:4558.

-27 Christoff K, Ream JM, Gabrieli JDE: Neural basis of spontaneous thought processes. Cortex 2004;40:1-9.

28 Giambra LM: A laboratory method for investigating influences on switching attention to task-unrelated imagery and thought. Conscious Cogn 1995;4:1-21.

29 McGuire PK, Paulescu E, Frackowiak RSJ, Frith CD: Brain activity during stimulus independent thought. Neuroreport 1996;7: 2095-2099.

30 Smallwood J, Obonsawin M, Heim D: Task unrelated thought: the role of distributed processing. Conscious Cogn 2003;2:169-189.

31 Teasdale JD, Dritschell BH, Taylor MJ, Proctor L, Lloyd CA, Nimmo-Smith I, Baddeley $\mathrm{AD}$ : Stimulus-independent thought depends upon central executive resources. Mem Cogn 1995;28:551-559.

32 Small DM, Gitelman DR, Gregory MD, Nobre AC, Parrish TB, Mesulam MM: The posterior cingulate and medial prefrontal cortex mediate the anticipatory allocation of spatial attention. Neuroimage 2003;18:633-641.

33 Wenzel R, Wobst P, Heekeren HH, Kwnong KK, Brandt SA, Kohl M, Obrig H, Dirnagl U, Villringer A: Saccadic suppression induces focal hypooxygenation in the occipital cortex. J Cereb Blood Flow Metab 2000;20: 1103-1110.

-34 Hock C, Villringer K, Muller-Spahn F, Wenzel R, Heekeren H, Schuh-Hofer S, Hofmann M, Minoshima S, Schwaiger M, Dirnagl U, Villringer A: Decrease in parietal cerebral hemoglobin oxygenation during perfor- mance of a verbal fluency task in patients with Alzheimer's disease monitored by means of near-infrared spectroscopy (NIRS) - correlation with simultaneous rCBF-PET measurements. Brain Res 1997;755:293303.

35 Sakatani K, Katayama Y, Yamamoto T, Suzuki S: Changes in cerebral blood oxygenation of the frontal lobe induced by direct electrical stimulation of thalamus and globus pallidus: a near-infrared spectroscopy study. J Neurol Neurosurg Psychiatry 1999;67:769773.

36 Fox PT, Raichle ME: Focal physiological uncoupling of cerebral blood flow and oxidative metabolism during somatosensory stimulation in human subjects. Proc Natl Acad Sci USA 1986;83:1140-1144.

37 Hoshi Y, Onoe H, Watanabe Y, Andersson J, Bergstrom M, Lilja A, Langstrom B, Tamura M: Non-synchronous behavior of neuronal activity, oxidative metabolism and blood supply during mental tasks in man. Neurosci Lett 1994;19:129-133.

38 Watanabe A, Matsuo K, Kato N, Kato T: Cerebrovascular response to cognitive tasks and hyperventilation measured by multi-channel near-infrared spectroscopy. J Neuropsychiatry Clin Neurosci 2003;15:442-449.

- 39 Herrmann MJ, Plichta MM, Ehlis A, Fallgatter AJ: Optical topography during a go-nogo task assessed with multi-channel near-infrared spectroscopy. Behav Brain Res 2005; 160 : 135-140.

40 Neisser U: Cognitive Psychology. New York, Appleton-Century-Crofts, 1967.

41 Gusnard DA, Raichle ME: Searching for a baseline: functional imaging and the resting human brain. Nat Rev Neurosci 2001;2:685694.

42 Raichle ME, MacLeod AM, Snyder AZ, Powers WJ, Gusnard DA, Shulman GL: A default mode of brain function. Proc Natl Acad Sci USA 2001;98:676-682.

43 Shulman GL, Fiez JA, Corbetta M, Buckner RL, Miezin FM, Raichle ME, Petersen SE: Common blood flow changes across visual tasks. II. Decreases in cerebral cortex. J Cogn Neurosci 1997;9:648-663.

44 Matsuo K, Onodera Y, Hamamoto T, Muraki K, Kato N, Kato T: Hypofrontality and microvascular dysregulation in remitted lateonset depression assessed by functional near-infrared spectroscopy. Neuroimage 2005;26:234-242.

-45 Jasdzewski G, Strangman G, Wagner J, Kwong KK, Poldrack RA, Boasa DA: Differences in the hemodynamic response to event-related motor and visual paradigms as measured by near-infrared spectroscopy. Neuroimage 2003;20:479-488.

-46 Schroeter ML, Zysset S, Kupka T, Kruggel F, Yves von Cramon D: Near-infrared spectroscopy can detect brain activity during a color-word matching Stroop task in an eventrelated design. Hum Brain Mapp 2002;17: 61-71. 